Algebra Univers. 61 (2009) 449-453

DOI $10.1007 / \mathrm{s} 00012-009-0028-9$

Published online November 18, 2009

Algebra Universalis

(C) 2009 The Author(s)

This article is published with open access at Springerlink.com

\title{
MacNeille completion and profinite completion can coincide on finitely generated modal algebras
}

\section{JACOB Vosmaer}

\begin{abstract}
Following Bezhanishvili and Vosmaer, we confirm a conjecture of Yde Venema by piecing together results from various authors. Specifically, we show that if $\mathbb{A}$ is a residually finite, finitely generated modal algebra such that $\operatorname{HSP}(\mathbb{A})$ has equationally definable principal congruences, then the profinite completion of $\mathbb{A}$ is isomorphic to its MacNeille completion, and $\diamond$ is smooth. Specific examples of such modal algebras are the free $\mathbf{K} 4$-algebra and the free PDL-algebra.
\end{abstract}

\section{Introduction}

In this paper we compare two mathematical constructions applied to modal algebras. The first is the MacNeille completion, which is an order-theoretic generalization of the construction of the reals from the rationals using Dedekind cuts [12]. It has been applied in logic to prove, for example, the completeness of predicate calculi [14]. The second is the profinite completion, which is a universal algebraic construction, transforming an algebra into a topological algebra endowed with a Stone (compact, Hausdorff, zero-dimensional) topology. This construction stems from Galois theory [15], but has more recently also been connected with lattice completions [3, 10, 19, 4].

This paper is a companion piece to [4]. In that paper, parallel versions of our Theorems 3.1 and 4.2 arise in a study of the connections between different completions of Heyting algebras, using Esakia duality. In light of the topological character of the profinite completion, in the present paper we will present topological algebra proofs instead. This establishes a strong connection with the body of work on canonicity [8] and MacNeille canonicity [17]. Another advantage of our present perspective is that we can show how the two main theorems pivot around an interaction between principal lattice filters and principal algebra congruences, which are in a one-to-one correspondence for Heyting algebras, but not for modal algebras. Finally, we will briefly mention some of the connections of our results to modal logic.

Presented by I. Hodkinson.

Received November 13, 2007; accepted in final form April 28, 2008.

2000 Mathematics Subject Classification: Primary: 06E25; Secondary: 06B23, 03B45, $22 \mathrm{~A} 30$.

Key words and phrases: modal algebra, MacNeille completion, profinite completion.

This research was supported by VICI grant 639.073.501 of the Netherlands Organization for Scientific Research (NWO). 


\section{Completions and topologies}

Let $\mathbb{B}=\langle B ; \wedge, \vee, \neg, 0,1\rangle$ be a Boolean algebra. Given $b \in B$ we write $b \downarrow=\{a \in B \mid a \leq b\}$ ( $b \uparrow$ is defined dually). We say $S \subseteq B$ is join-dense in $\mathbb{B}$ iff, for every $a \in B, a=\bigvee(a \downarrow \cap S)$ (meet-density is defined dually). A completion of a lattice $\mathbb{B}$ is a pair $(m, \mathbb{C})$, where $m: \mathbb{B} \hookrightarrow \mathbb{C}$ is a lattice embedding into a complete lattice $\mathbb{C}$. Completions $(m, \mathbb{C})$ and $(k, \mathbb{D})$ of $\mathbb{B}$ are isomorphic if $g m=k$ for some lattice isomorphism $g: \mathbb{C} \rightarrow \mathbb{D}$. If $(m, \mathbb{C})$ is a completion of $\mathbb{B}$, let $\rho_{\mathbb{B}}$ be the topology on $C$ generated by basis $\{[m(a), m(b)] \mid a, b \in B\}$ (where $[x, y]=\{z \in C \mid x \leq z \leq y\}$ ). By $\gamma_{\mathbb{B}}^{\downarrow}, \gamma_{\mathbb{B}}^{\uparrow}$ and $\gamma_{\mathbb{B}}$ we denote the Scott topology, the dual Scott topology, and the biScott topology on $\mathbb{B}$ respectively. Let At $\mathbb{B}$ be the (possibly empty) set of atoms of $\mathbb{B}$, and let $\operatorname{At}_{\omega} \mathbb{B}$ be the set of all finite joins of atoms of $\mathbb{B}$. Then $\iota_{\mathbb{B}}$ is the topology generated by the basis $\left\{[a, \neg b] \mid a, b \in \mathrm{At}_{\omega} \mathbb{B}\right\}$. By $[8$, Section 2$], \iota_{\mathbb{B}}=\gamma_{\mathbb{B}}$ if $\mathbb{B}$ is complete and atomic.

The MacNeille completion [2] of a Boolean algebra $\mathbb{B}$ is defined up to isomorphism as a completion $(m, \mathbb{C})$ such that $m[B]$ is join-dense in $\mathbb{C}$ (by $[5$, Theorem V-27] $\mathbb{C}$ is then also a Boolean algebra). We denote the MacNeille completion of $\mathbb{B}$ by $\overline{\mathbb{B}}$. Alternatively $[17$, Theorem 4.5$], \overline{\mathbb{B}}$ can be characterized up to isomorphism as a completion $(m, \mathbb{C})$ of $\mathbb{B}$ such that $\left\langle C, \rho_{\mathbb{B}}\right\rangle$ is Hausdorff. If $f: \mathbb{B} \rightarrow \mathbb{C}$ is an order-preserving map between Boolean algebras, then $f^{\circ}: \overline{\mathbb{B}} \rightarrow \overline{\mathbb{C}}$, defined by $f^{\circ}: x \mapsto \bigvee\left\{f(a) \mid m_{\mathbb{B}}(a) \leq x\right\}$, is the lower extension of $f$. The upper extension $f^{\bullet}$ is defined dually. Alternatively [17, Section 5], $f^{\circ}$ is the (pointwise) largest $\left(\rho_{\mathbb{B}}, \gamma_{\overline{\mathbb{C}}}^{\downarrow}\right)$-continuous extension of $f$, and $f^{\bullet}$ is the smallest $\left(\rho_{\mathbb{B}}, \gamma_{\overline{\mathbb{C}}}^{\uparrow}\right)$-continuous extension of $f$. We say $f$ is smooth if $f^{\circ}=f^{\bullet}$.

Given a modal algebra $\mathbb{A}=\langle A ; \diamond\rangle$, let $\Phi_{\mathbb{A}}:=\{\theta \in$ Con $\mathbb{A} \mid \mathbb{A} / \theta$ is finite $\}$. We say $\mathbb{A}$ is residually finite if, for all $a, b \in \mathbb{A}$ with $a \neq b$, there exists $\theta \in \Phi_{\mathbb{A}}$ such that $a / \theta \neq b / \theta$. The inverse system $\left\langle\{\mathbb{A} / \theta\}_{\theta \in \Phi_{\mathbb{A}}}, f_{\theta \psi}\right\rangle$, where $f_{\theta \psi}: \mathbb{A} / \theta \rightarrow$ $\mathbb{A} / \psi$ (for all $\theta, \psi \in \Phi_{\mathbb{A}}$ such that $\theta \subseteq \psi$ ) is defined by $f_{\theta \psi}: a / \theta \mapsto a / \psi$, has a projective limit

$\hat{\mathbb{A}}=\left\{\alpha \in \prod_{\Phi_{\mathbb{A}}} \mathbb{A} / \theta \mid \forall \theta, \psi \in \Phi_{\mathbb{A}}\right.$ with $\theta \subseteq \psi$, if $\alpha(\theta)=a / \theta$ then $\left.\alpha(\psi)=a / \psi\right\}$.

The map $\mu: \mathbb{A} \rightarrow \hat{\mathbb{A}}$, defined by $\mu: a \mapsto(a / \theta)_{\theta \in \Phi_{\mathbb{A}}}$, is a modal algebra homomorphism which is injective iff $\mathbb{A}$ is residually finite. We call $\hat{\mathbb{A}}$ the profinite completion of $\mathbb{A}[15]$. Since $\hat{\mathbb{A}}$ is a complete lattice [10], it follows that $(\mu, \hat{\mathbb{A}})$ is a completion of $\mathbb{A}$ iff $\mathbb{A}$ is residually finite. If we define the discrete topology on each $\mathbb{A} / \theta, \hat{\mathbb{A}}$ inherits a topology $\tau_{\hat{\mathbb{A}}}$ as a closed subspace of the product $\prod_{\Phi_{\mathbb{A}}} \mathbb{A} / \theta$. Now $\left\langle\hat{A}, \tau_{\hat{\mathbb{A}}}\right\rangle$ is a Stone space [3, Section 2], and in particular $\widehat{\diamond}: \hat{\mathbb{A}} \rightarrow \hat{\mathbb{A}}$ is $\left(\tau_{\hat{\mathbb{A}}}, \tau_{\hat{\mathbb{A}}}\right)$-continuous $[1]$.

Lemma 2.1. If $\mathbb{A}$ is a Boolean algebra expansion, then $\tau_{\hat{\mathbb{A}}}=\iota_{\hat{\mathbb{A}}}=\gamma_{\hat{\mathbb{A}}}$.

Proof. Since $\left\langle\hat{\mathbb{A}}, \tau_{\hat{\mathbb{A}}}\right\rangle$ is a compact Hausdorff topological lattice, it follows by $[9$, Corollary VII-2.3] that $\tau_{\hat{\mathbb{A}}}=\gamma_{\hat{\mathbb{A}}}$. Since $\hat{\mathbb{A}}$ is also a complete, atomic Boolean algebra $[3,19]$, we know that $\iota_{\hat{\mathbb{A}}}=\gamma_{\hat{\mathbb{A}}}[8$, Section 2]. 


\section{Comparing profinite completion and MacNeille completion}

Theorem 3.1 (cf. [4, Theorem 4.12]). Let $\mathbb{A}$ be a modal algebra. The following are equivalent:

(1) the profinite completion $(\mu, \hat{\mathbb{A}})$ is the MacNeille completion of $\mathbb{A}$, and $\diamond$ is smooth;

(2) $\mathbb{A}$ is residually finite and, for every $\theta \in \Phi_{\mathbb{A}}, 1 / \theta$ is a principal lattice filter.

Proof. If $(\mu, \hat{\mathbb{A}})$ is the MacNeille completion of $\mathbb{A}$, then $\mu: \mathbb{A} \rightarrow \widehat{\mathbb{A}}$ must be injective, so that $\mathbb{A}$ is residually finite (see above). Let $\theta \in \Phi_{\mathbb{A}}$; then it follows from the definition of $\hat{\mathbb{A}}$ that the projection $\pi_{\theta}: \hat{\mathbb{A}} \rightarrow \mathbb{A} / \theta$ commutes with $\mu$ and the natural map $a \mapsto a / \theta$; i.e., $a / \theta=\pi_{\theta} \mu(a)$. By [3, Lemma 2.7], $\pi_{\theta}^{-1}(1 / \theta)$ is a closed principal filter of $\widehat{\mathbb{A}}$; say $\pi_{\theta}^{-1}(1 / \theta)=\alpha \uparrow$. Because of the correspondence between modal filters and modal congruences [18, Theorem 29], $\alpha \uparrow$ completely characterizes $\mathbb{A} / \theta$ in the following sense: $\mathbb{A} / \theta \cong[0, \alpha]_{\hat{\mathbb{A}}}$ as a bounded lattice [7, Exercise 4.12]. This implies that $\alpha \downarrow$ is finite. Since $(\mu, \hat{\mathbb{A}})$ is the MacNeille completion of $\mathbb{A}, \mu[\mathbb{A}]$ is join-dense in $\hat{\mathbb{A}}$, so by finiteness of $\alpha \downarrow$, there must exist $a \in \mathbb{A}$ such that $\mu(a)=\alpha$. Now $b / \theta=1 / \theta$ iff $\mu(b) \in \pi_{\theta}^{-1}(1 / \theta)=\alpha \uparrow=\mu(a) \uparrow$ iff $b \geq a$, so $1 / \theta=a \uparrow$ is a principal lattice filter.

Conversely, if $\mathbb{A}$ is residually finite, then $\mu: \mathbb{A} \rightarrow \hat{\mathbb{A}}$ is injective, so $(\mu, \hat{\mathbb{A}})$ is a completion of $\mathbb{A}$. To show that $(\mu, \hat{\mathbb{A}})$ is the MacNeille completion of $\mathbb{A}$, we will consider the different topologies on $\hat{\mathbb{A}}$. We first show that $\operatorname{At} \hat{\mathbb{A}} \subseteq \mu[\mathbb{A}]$. If $\alpha \in$ At $\hat{\mathbb{A}}$, there must be some $\theta \in \Phi_{\mathbb{A}}$ such that $\alpha(\theta) \in \operatorname{At} \mathbb{A} / \theta$. Because $1 / \theta$ is a principal lattice filter $c \uparrow$, we know that $\mathbb{A} / \theta \cong[0, c]_{\mathbb{A}}$ as a bounded lattice, so there must be some $a \leq c$ with $a \in$ At $\mathbb{A}$ and $a / \theta=\alpha(\theta)$. But then $\mu(a)=\alpha$. It follows that At $\hat{\mathbb{A}} \subseteq \mu[\mathbb{A}]$, whence $\iota \subseteq \rho$. Since $\iota$ is Hausdorff, so is $\rho$. Using [17, Theorem 4.5] we can thus conclude that, as far as the Boolean substructure of $\mathbb{A}$ is concerned, $(\mu, \hat{\mathbb{A}})$ is the MacNeille completion of $\mathbb{A}$. Now to show that $\diamond$ is smooth, remember that $\widehat{\diamond}: \widehat{\mathbb{A}} \rightarrow \widehat{\mathbb{A}}$ is $(\tau, \tau)$-continuous. Since $\tau=\iota=\gamma$ by Lemma 2.1 and $\iota \subseteq \rho$, it follows that $\widehat{\diamond}$ is $(\rho, \gamma)$-continuous. But then since $\gamma^{\downarrow}, \gamma^{\uparrow} \subseteq \gamma$, it follows by [17, Proposition 5.9] that $\diamond^{\bullet} \leq \widehat{\diamond} \leq \diamond^{\circ}$. Since also $\diamond^{\circ} \leq \diamond^{\bullet}[17$, Proposition 5.6], it follows that $\diamond$ is smooth.

Note that the theorem above also admits a third equivalent condition, characterizing the dual space of $\mathbb{A}$. This perspective is further explored in [4].

\section{Finitely generated modal algebras with EDPC}

Having equationally definable principal congruences (EDPC) is a strong meta-logical property of varieties of algebras that coincides with, for example, the existence of a deduction theorem or of a master modality $[6,11]$ for the modal logic corresponding to a variety of modal algebras. Examples of such logics are logics of bounded depth, $n$-transitive logics such as $\mathbf{K} \mathbf{4}$, or regular test-free PDL with finitely many basic programs. 
Lemma 4.1 (Proposition 3.4.3 of [11]). Let $\mathcal{V}$ be a variety of modal algebras. $\mathcal{V}$ has EDPC iff every principal modal filter of an algebra in $\mathcal{V}$ is a principal lattice filter.

Note that the hypotheses below strongly resemble those of [20, Theorem 4]. Theorem 4.2 (cf. [4, Corollary 4.5.3]). If $\mathbb{A}$ is a residually finite, finitely generated modal algebra such that $\operatorname{HSP}(\mathbb{A})$ has equationally definable principal congruences, then the profinite completion $(\mu, \hat{\mathbb{A}})$ is the MacNeille completion of $\mathbb{A}$ and $\diamond$ is smooth.

Proof. Since $\mathbb{A}$ is a finitely generated algebra, every $\theta \in \Phi_{\mathbb{A}}$ is compact $[16$, Theorem 1]. As is remarked in [20], every compact congruence of a modal algebra is principal, so under our hypotheses, every $\theta \in \Phi_{\mathbb{A}}$ is principal. Now since $\operatorname{HSP}(\mathbb{A})$ has $\operatorname{EDPC}$, Lemma 4.1 tells us that $1 / \theta$ is a principal lattice filter for all $\theta \in \Phi_{\mathbb{A}}$, so by Theorem 3.1, $(\mu, \hat{\mathbb{A}})$ is the MacNeille completion of $\mathbb{A}$ and $\diamond$ is smooth.

Note that the EDPC clause in the theorem above is suppressed in the Heyting algebra case [4], because every variety of Heyting algebras has EDPC.

The conditions of Theorem 4.2 above are sufficient; what about necessity? From Theorem 3.1 we know that it is necessary that $\mathbb{A}$ is residually finite. Moreover, in light of [19, Section 3.3], we know that it is necessary for Theorem 4.2 that $\mathbb{A}$ is atomic. This helps us to find counterexamples to the theorem if we remove the requirements of being finitely generated or having EDPC. For instance, let $\mathbb{A}$ be the free algebra on one generator for the modal logic $\mathbf{T}$ (the logic of reflexive Kripke frames). Then [20, Corollary 7: Example 1] tells us that $\mathbb{A}$ is residually finite and finitely generated but not atomic. This shows us that being residually finite and finitely generated is not sufficient for the conclusion of Theorem 4.2. Alternatively, the free transitive modal algebra on $\omega$ generators is an example of a residually finite modal algebra $\mathbb{A}$, generating a variety with EDPC, such that $\mathbb{A}$ is not atomic. In summary, residual finiteness is necessary for Theorem 4.2, and if we remove either of the other two conditions, we can find non-atomic counterexamples.

Corollary 4.3. Let $\mathbb{A}$ be a finitely generated free algebra for $\mathbf{K} 4$ or $\mathbf{P D L}$. Then the MacNeille completion and the profinite completion of $\mathbb{A}$ are the same and $\diamond$ is smooth.

Proof. Let $\mathbf{L}$ be either $\mathbf{K} 4$ or $\mathbf{P D L}$ and let $\mathcal{V}$ be the variety corresponding to $\mathbf{L}$. Since $\mathbf{L}$ has the finite model property, $\mathcal{V}$ is generated by its finite members. By [13, Theorem IV-14.4], this implies that $\mathbb{A}$, being a finitely generated free algebra for $\mathcal{V}$, is residually finite. Using the fact that $\mathbf{L}$ has a master modality, it follows that we can apply Theorem 4.2.

Acknowledgements. The author would like to thank Yde Venema, who suggested that Theorem 4.2 might be true. Additionally, the author is grateful to the Editor and the Referee for their criticisms and suggestions. 


\section{REFERENCES}

[1] Banaschewski, B.: On profinite universal algebras. In: Novák, J. (ed.) Proceedings of the Third Prague Topological Symposium, pp. 51-62, September 1971

[2] Banaschewski, B., Bruns, G.: Categorical characterization of the MacNeille completion. Arch. Math. (Basel) 18, 369-377 (1967)

[3] Bezhanishvili, G., Gehrke, M., Mines, R., Morandi, P.J.: Profinite completions and canonical extensions of Heyting algebras. Order 23, 143-161 (2006)

[4] Bezhanishvili, G., Vosmaer, J.: Comparison of MacNeille, canonical and profinite completions. Order 25, 299-320 (2008)

[5] Birkhoff, G.: Lattice Theory, 3rd edn. American Mathematical Society (1967)

[6] Blok, W., Pigozzi, D.: On the structure of varieties with equationally definable principal congruences III. Algebra Universalis 32, 545-608 (1994)

[7] Davey, B.A., Priestley, H.A.: Introduction to Lattices and Order, 2nd edn. Cambridge University Press (2002)

[8] Gehrke, M., Jónsson, B.: Bounded distributive lattice expansions. Math. Scand. 94, $13-45$ (2004)

[9] Gierz, G., Hofmann, K.H., Keimel, K., Lawson, J.D., Mislove, M., Scott, D.S.: A Compendium of Continuous Lattices. Springer (1980)

[10] Harding, J.: On profinite completions and canonical extensions. Algebra Universalis 55, 293-296 (2006)

[11] Kracht, M.: Tools and Techniques in Modal Logic. Elsevier (1999)

[12] MacNeille, H.M.: Partially ordered sets. Trans. Amer. Math. Soc. 42, 416-160 (1927)

[13] Mal'cev, A.I.: Algebraic Systems. Springer (1973)

[14] Rasiowa, H., Sikorski, R.: A proof of the completeness theorem of Gödel. Fund. Math. 37, 193-200 (1950)

[15] Ribes, L., Zalesskii, P.: Profinite Groups. Springer (2000)

[16] Rival, I., Sands, B.: A note on the congruence lattice of a finitely generated algebra. Proc. Amer. Math. Soc. 72, 451-455 (1978)

[17] Theunissen, M., Venema, Y.: MacNeille completions of lattice expansions. Algebra Universalis 57, 143-193 (2007)

[18] Venema, Y.: Algebras and Coalgebras. In: Blackburn, P., et al. (eds.) Handbook of Modal Logic. Elsevier (2007)

[19] Vosmaer, J.: Connecting the profinite completion and the canonical extension using duality. Master's thesis, University of Amsterdam (2006)

[20] Wolter, F.: A note on atoms in polymodal algebras. Algebra Universalis 37, 334-341 (1997)

\section{Jacob Vosmaer}

Postbus 94242, 1090 GE Amsterdam, The Netherlands

e-mail: jvosmaer@science.uva.nl

Open Access This article is distributed under the terms of the Creative Commons Attribution Noncommercial License which permits any noncommercial use, distribution, and reproduction in any medium, provided the original author(s) and source are credited. 